\title{
Multiple Burr Hole as an Alternative Treatment for Large Scalp Defect
}

Background: Scalp defect with exposed bones is a serious injury that is often occurred after electrical burn injury. The coverage treatment may vary from

Priscilla Valentin N. Iswinarno Doso Saputro local flap to free flap. Coverage becomes a major problem when flaps fails or is contraindicated and resulting in further morbidity. In this paper we report 1 patient with large scalp defect after electrical burn injury.

Methods : In this study, we report 1 patient with large scalp defect after electrical burn, which treated in our department. Fourteen days after the electrical burn injury, surgical debridement was done by the plastic surgeon, and multiple burr holes were made by the neurosurgeon in the nonviable bone. The distance between each burr holes was $15 \mathrm{~mm}$. The defect was keep moist with absorbent. The scalp defects were treated with soft tissue debridement every 3 days in the policlinics to keep the wound presented viable. To date, the wound already treated for 90 days.

Results : The granulation tissue appears in between the burr holes within 14 days. After the defect was covered with granulation tissues, a further closure such as skin graft application was necessary. No postoperative infection, osteomyelitis, or cranial bone sequestration was reported in this case. Conclusions:

Multiple cranial burr holes can be chosen as an alternative treatment for large scalp defect. These technique, allow the formation of a vascular bed suitable for skin grafting. This technique is safe, swift and effective for cases of extensive exposure for the skull bone expose of the periosteum following trauma.

Keyword: Burr holes, electric burn, scalp
$\mathrm{S}$ calp defect with exposed bones is a serious injury that is often occurred after electrical burn injury. The coverage treatment may vary from local flap to free flap. Coverage becomes a major problem when flaps fails or is contraindicated and resulting in further morbidity. When scalp reconstruction is difficult to perform either by free flap due to old age and multiple medical problems or by local flap due to too large a defect, an alternative method of burr holes can be chosen for inducing the granulation tissue.

However, when performing a closure defect to bare calvarial bone, it is difficult to be taken. Therefore, to ensure that a skin graft or flap is taken, the outer table of the necrotic calvarial bone is removed in the first place to allow the granulation tissue to grow. Subsequently, the skin graft or flap is performed as a second stage procedure. In scalp wounds with adequate vascularity, some literature has reported cases of immediate reconstruction with skin grafting without time in between for wound bed preparation. But in cases where poor vascularity is highly suspected due to electrical burn, the burr holes treatment was an alternative to inducing the granulation tissue. However, two-stage approach is time consuming and raises the overall morbidity and complication rate, such as making the graft vulnerable to infection and causing the osteomyelitis.

\section{Material \& Methods}

In this study, we report 1 patient with large scalp defect after electrical burn injury. After surgical debridement, this patient was underwent the burring of external cortex of

Disclosure: The authors herely declare they have no financial interest in the information discussed in this article 
the skull with the help of burrs under general anesthesia. The punctured burr hole was done by the neurosurgeon.

The punctured on the outer table of cortical bone caused exposure of diplopic space. The hole was made to the vascular diploe making a series of squares of approximately $1.5 \mathrm{~cm}$ dimension. The depth of bony penetration using the hand drill is such more accurately controlled than with the standard technique using an osteotome and thus the risk of exposing appreciable areas of dura mater minimized. From the second postoperative day, the wound was daily dressed using the non-adherent, antibiotic coated tulle-gras to keep it moist.

To this end, we present the clinical outcomes of one patient with the exposed skull with through long-term follow-ups over at least 13 months after the procedure.

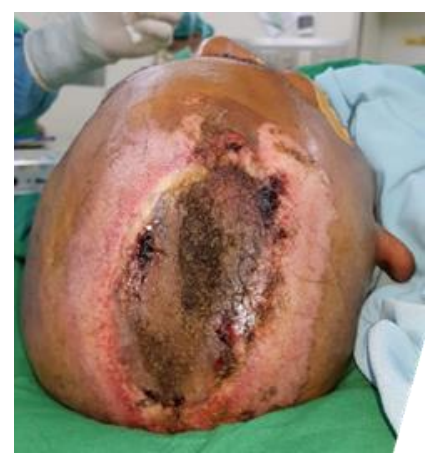

Figure 1. 49 years old male with $15 \times 6 \mathrm{~cm}$ non viable cranial bone exposed. Before surgical debridement.

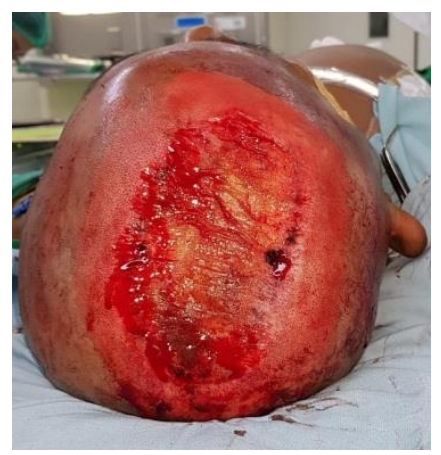

Figure 2. The exposed calvaria after surgical debridement

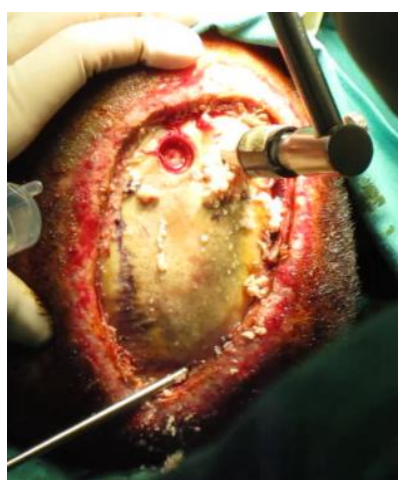

Figure 3. Burrholes were done by the neurosurgeon

\section{Discussion}

Conservative management that awaits spontaneous sequestration in the outermost table may take several months to occur and is aesthetically unsatisfactory for patients and relatives and requires a long surgical dressing.

Expanded or transposed scalp flaps may not be feasible where large areas of the calvarium have been exposed and in particular the recently introduced techniques of tissue expansion, as described by Argenta and his colleagues (l), are not suitable in proximity to an infected burn wound.

The multiple burr holes filled with fibrous tissue and the contour of the skull was maintained. The exposed calvaria, must be covered by living tissue or bone will be discarded as sequestrum. Cancellous underlying reaction bone, pretty much blood supply, is to produce a layer of granulation tissue that invites open bones and separates the dead sequestrum cortex as part or whole. This granulation network, in the company with osteoclasts, it is able to absorb sequestrum and in the process of growing repeatedly, so this is the basis for the survival of free grafts.

The effect of multiple drilling allows access to the granulation tissue to reach the surface of the bone and spread over the cortex. This compares unfavourably with decortication, which does attempt to expose the whole area and allow granulations to sprout through multiple bleeding points. 
There is reason to believe, however, that the presence of granulation is more than one open areas of cortical bone will maintain their viability and act, in part, as periosteal cover.

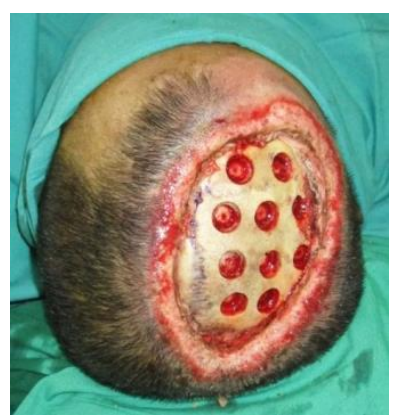

Figure 4. Multiple burr holes were done

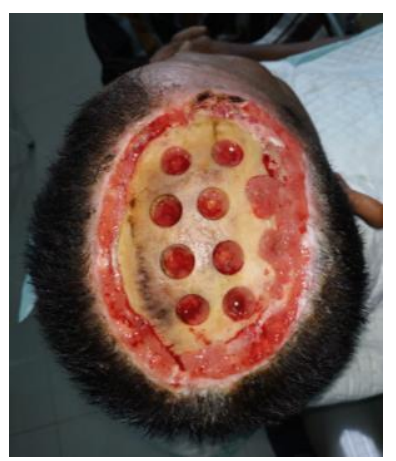

Figure 5.18 days after the operation. Few granulation tissue was indicated.

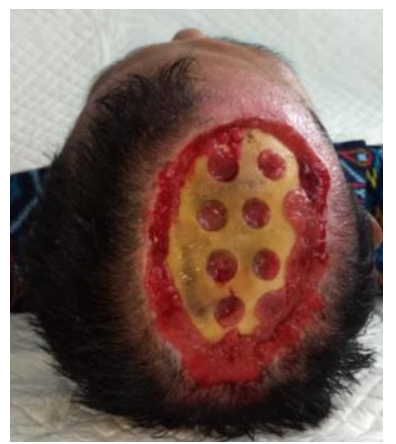

Figure 6. 38 days after the operation. The granulation tissue was indicated.

This technique has been found useful in rapidly resurfacing exposed outer table denuded of periosteum in a variety of conditions involving burns in the acute and long-term stages and in cases where the outer table is left exposed following ablation of scalp. In the other hand, further defect closure such as skin grafts or flaps was necessary in order to closed the defect.

No postoperative infection, osteomyelitis, or cranial bone sequestration occurred in this cases.This graph shows that traffic accidents are the most common cause of palate fracture due to the lack of public awareness of driving compliance. On the other hand, workplace and household accidents are second, followed by violence, the majority of which are caused by criminal cases.

\section{Conclusion}

Multiple cranial burr holes can be chosen as an alternative treatment for large scalp defect. These technique, allow the formation of a vascular bed suitable for skin grafting. Even with moderately delayed management of electrical burns, partial excision of the necrotic bone with burr holes and flap coverage appears to be adequate. This technique is safe, swift and effective for cases of extensive exposure for the skull bone expose of the periosteum following trauma.

\section{Reference}

1. Pitkanen JM, Al-Qattan MM, Russel NA (2000) Immediate coverage of exposed, denuded cranial bone with split-thickness skin grafts. Ann Plast Surg 45:118-121

2. Tamas C, Popa L, Turliuc D, Morosanu C, Lazar M. Surgical reconstruction in scalp defects. Clinics of Plastic and Reconstructive Surgery. 2005. 1(2); 83-86)

3. Lisa M. Earnest, Patrick J. Byrne. Scalp Reconstruction. Facial plastic surgery clinics of North America. May 2005 13(2): 345-353

4. Fan J, Yang P. Aesthetic reconstruction of burn alopecia by using expanded hairbearing scalp flaps. Aesthetic Plast Surg.1997 Nov-Dec; 21(6): 440-4.).

5. Leedy JE, Janis JE, Rohrich RJ. Reconstruction of acquired scalp defects: an algorithmic approach. Plast Reconstr Surg. 2005 Sep 15; 116(4): 54e-72e.

6. Newman MI, Hanasono MM, Disa JJ, Cordeiro PG, Mehrara BJ. Scalp reconstruction: a 15year experience. Ann Plast Surg. 2004 May; 52(5): 501-6. 